\title{
Placental PHLDA2 expression is increased in cases of fetal growth restriction following reduced fetal movements
}

\author{
Anna Bugge Janssen ${ }^{1}$, Simon J. Tunster ${ }^{1}$, Alexander E. P. Heazell ${ }^{2}$ and Rosalind M. John ${ }^{1 *}$ (D
}

\begin{abstract}
Background: Maternal perception of reduced fetal movements (RFM) is associated with increased risk of fetal growth restriction (FGR) and stillbirth, mediated by placental insufficiency. The maternally expressed imprinted gene PHLDA2 controls fetal growth, placental development and placental lactogen production in a mouse model. A number of studies have also demonstrated abnormally elevated placental PHLDA2 expression in human growth restricted pregnancies. This study examined whether PHLDA2 was aberrantly expressed in placentas of RFM pregnancies resulting in delivery of an FGR infant and explored a possible relationship between PHLDA2 expression and placental lactogen release from the human placenta.
\end{abstract}

Methods: Villous trophoblast samples were obtained from a cohort of women reporting RFM $(N=109)$ and PHLDA2 gene expression analysed. hPL levels were assayed in the maternal serum ( $N=74)$.

Results: Placental PHLDA2 expression was significantly 2.3 fold higher in RFM pregnancies resulting in delivery of an infant with FGR $(p<0.01)$, with highest levels of PHLDA2 expression in the most severe cases. Placental PHLDA2 expression was associated with maternal serum hPL levels $(r=-0.30, p=0.008, n=74)$ although this failed to reach statistical significance in multiple linear regression analysis controlling for birth weight $(p=0.07)$.

Conclusions: These results further highlight a role for placental PHLDA2 in poor perinatal outcomes, specifically FGR associated with RFM. Furthermore, this study suggests a potential relationship between placental PHLDA2

expression and hPL production by the placenta, an association that requires further investigation in a larger cohort.

Keywords: Reduced fetal movements, Stillbirth, Fetal growth restriction, Placenta, PHLDA2

\section{Background}

Maternal perception of reduced fetal movements (RFM) is associated with an increased risk of poor perinatal outcomes such as fetal growth restriction (FGR), preterm birth, fetal distress, and stillbirth [1-7]. Placental insufficiency, the reduced ability of the placenta to supply nutrients and oxygen to the fetus, could explain the cooccurrence of RFM and FGR [8]. Placental insufficiency is thought to primarily result in asymmetric FGR with prolonged exposure resulting in RFM (as a mechanism for conserving energy for vital functions) and, if this remains undetected, stillbirth [8]. Indeed, altered placental structure and function has been reported in RFM pregnancies

\footnotetext{
* Correspondence: JohnRM@cardiff.ac.uk

${ }^{1}$ Cardiff School of Biosciences, Cardiff University, Cardiff, Wales CF10 3AX, UK Full list of author information is available at the end of the article
}

[9-12]. For example, RFM placentas are smaller, lighter and exhibit a specific reduction in the syncytiotrophoblast layer of the placenta [9], which synthesises hormones such as human placental lactogen (hPL) for release into the maternal circulation [13].

Imprinted genes are monoallelically expressed with expression depending on the parent of origin [14]. These genes have well-established roles in controlling fetal growth and placental development, and are regulated by epigenetic marks that may respond to environmental stimuli [15-17]. A subset of imprinted genes also converge on the endocrine lineages of the mouse placenta to regulate placental hormone production [18]. The maternally expressed imprinted gene PLECKSTRIN HOMOLOGY-LIKE DOMAIN FAMILY A MEMBER 2 (PHLDA2) exemplifies all of these functions. Importantly, abnormally elevated placental PHLDA2 
expression has been associated with FGR and/or low birth weight in a number of studies (reviewed in [16]). Modelling similar overexpression of Phlda2 in a mouse model results in FGR [19, 20], inferring that the association observed in human pregnancies is causal. Moreover, the growth restriction which occurs in response to Phlda2 overexpression is late onset and asymmetric, followed by rapid postnatal catch up growth [20], which is typical of human pregnancies complicated by placental insufficiency. The Phlda 2 overexpression mouse model also exhibits stunted placental growth with a specific reduction in a key endocrine lineage and impaired placental lactogen production [19, 21], consistent with the altered endocrine function observed in human RFM placentas $[9,12]$.

In this study we analysed PHLDA2 expression in a cohort of women reporting RFM $(N=109)$. We hypothesised that placental PHLDA2 expression is specifically increased in RFM pregnancies complicated by FGR $(N=20)$. We also analysed maternal serum hPL levels $(N=74)$ in the RFM cohort to explore the potential link between PHLDA2 expression and placental lactogen in the human placenta.

\section{Methods}

\section{Participant recruitment}

Study participants included women delivering within one week of presentation with maternal perception of
RFM (after 28 weeks gestation) [2, 9]. Written informed consent was obtained from the participants and the study was approved by Oldham and Greater Manchester North Research Ethics Committees (REC no. 08/1011/83 and $11 / \mathrm{NW} / 0664)$. Fetal growth restriction was defined as customised birth weight centile $<10^{\text {th }}[2]$. Participant demographics are shown in Table 1.

\section{Sample collection}

Villous trophoblast samples were obtained within $30 \mathrm{~min}$ of delivery [9]. As intraplacental variation in PHLDA2 expression has previously been demonstrated [22], samples were consistently taken midway between the cord insertion and distal edge. Maternal venous serum samples were obtained on admission $(N=74)$ and hPL levels measured by ELISA (Immunodiagnostic systems, Boldon, UK) as previously described [2].

\section{Gene expression analysis}

Total RNA was extracted from the placental tissue samples using GenElute Mammalian Total RNA Miniprep Kit (Sigma-Aldrich, Dorset, UK). $5 \mu \mathrm{g}$ of RNA was reverse transcribed using M-MuLV reverse transcriptase (Promega, Southampton, UK) with random hexamers, according to manufacturer's instructions. Quantitative RT-PCR was performed using Chromo 4 Four Colour

Table 1 Participant demographics of RFM participants $(N=109)$ Mean (SD)/Range or Number (\%)

\begin{tabular}{|c|c|c|c|}
\hline \multicolumn{4}{|l|}{ Birth outcomes } \\
\hline Fetal sex & & Ethnicity & \\
\hline Male & $56(51 \%)$ & Caucasian & $73(67 \%)$ \\
\hline Female & $53(49 \%)$ & African/Afro-Caribbean & $10(9 \%)$ \\
\hline Gestational age (days) & $278(13) / 211-295$ & Indian/Pakistani/Bangladeshi & $21(19 \%)$ \\
\hline Birth weight (g) & $3306(599) / 850-4680$ & Other & $5(5 \%)$ \\
\hline Custom birth weight centile & $40(29) / 0-100$ & Parity & $1(1.22) / 0-7$ \\
\hline Placental Weight (g) & $587(127) / 353-854$ & Smoking during Pregnancy & \\
\hline Apgar Scores (1 min) & $9(1.66) / 0-10$ & Yes & $17(16 \%)$ \\
\hline Apgar Scores (5 min) & $10(1.39) / 0-10$ & No & $92(84 \%)$ \\
\hline Mode of Delivery & & Alcohol consumption & \\
\hline SVD & $61(56 \%)$ & None & 107 (98 \%) \\
\hline ELCS & $10(9 \%)$ & 1-5 units/week & $2(2 \%)$ \\
\hline EMCS & $14(13 \%)$ & Obstetric complications & \\
\hline Instrumental & $24(22 \%)$ & None & 84 (77 \%) \\
\hline Maternal characteristics & & Preeclampsia/PIH/Proteinuria & $5(5 \%)$ \\
\hline Age (years) & $29(5.81) / 17-46$ & Repeat RFM & $10(9 \%)$ \\
\hline \multirow[t]{3}{*}{ Maternal BMI } & $25.78(5.26) / 17-46$ & Oligohydramnios & $1(1 \%)$ \\
\hline & & Suspected growth restriction & $3(3 \%)$ \\
\hline & & Other & $4(4 \%)$ \\
\hline
\end{tabular}


Real Time Detector (MJ Research) in a $20 \mu \mathrm{l}$ reaction containing $5 \mu \mathrm{l}$ of cDNA (diluted 1 in 50), 1X Buffer $2 \mathrm{mM} \mathrm{MgCl}$, $2 \mathrm{mM}$ dNTPs, 0.65 Units Taq (Fermentas (Thermo), Loughborough, UK), $1 \mu \mathrm{M}$ of each primer (Sigma-Aldrich, Dorset, UK) and 0.12X Sybr Green (Invitrogen, Paisley, UK). All samples were run in triplicate and duplicate plates were performed. Conditions for amplification were: 1) $15 \mathrm{~min}$ at $94{ }^{\circ} \mathrm{C}$, 2) $30 \mathrm{~s}$ at $94{ }^{\circ} \mathrm{C}$, 3) $30 \mathrm{~s}$ at $60{ }^{\circ} \mathrm{C}$, 4) $30 \mathrm{~s}$ at $72{ }^{\circ} \mathrm{C}$ and 5) $30 \mathrm{~s} 75{ }^{\circ} \mathrm{C}$, with steps 2-5 repeated for a total of 40 cycles. Melt Curve was performed from 70 to $94{ }^{\circ} \mathrm{C}$, reading every $0.5{ }^{\circ} \mathrm{C}$ and holding for $2 \mathrm{~s}$. Primer sequences were as follows: YWHAZ forward: TTCTTGATCCCCAATGCTTC \& reverse: AGTTAAGGGCCAGACCCAGT, PHLDA2 forward: GAGCGCACGGGCAAGTA \& reverse: CAGCG GAAGTCGATCTCCTT [23] and $L 19$ forward: CCAAC TCCCGTCAGCAGATC \& reverse: CAAGGTGTTTTT CCGGCATC [24].

\section{Statistical analysis}

Gene expression data is presented as the $\Delta C T$ (target gene expression relative to the housekeeping gene YWHAZ) and as the fold change in expression, calculated using the $2^{-\triangle \Delta C T}$ method [25]. The geometric mean of two housekeeping genes, YWHAZ and L19, was used to confirm results obtained using the single housekeeping gene, YWHAZ, for a subset of samples. Parametric statistical tests were used to analyse normally distributed data. The effect of potential confounders (infant birth weight, gender and gestational age) was examined using multiple linear regression analysis. To ease interpretation, $\triangle \mathrm{CT}$ values have been inverted $[x(-1)]$ such that lower values represent decreasing gene expression.

\section{Results}

Placental PHLDA2 expression was significantly 2.3 fold higher in RFM pregnancies resulting in delivery of a growth restricted compared with a normal birth weight infant (Fig. 1a). Results remained statistically significant when comparing normalisation to a single housekeeping gene $Y W H A Z$ with the geometric mean of $Y W H A Z$ and $L 19$ expression in a subset of samples (Additional file 1: Figure S1).

When participants were further classified according to severity of growth restriction, PHLDA2 expression was significantly 1.7 fold higher in cases with custom birth weight centile $<10^{\text {th }}(p=0.01, n=12$. v. 82) and 3.2 fold higher in cases with custom birth weight centile $<3^{\text {rd }}(p<0.001, n=8$ v. 82). Placental PHLDA2 expression was also significantly inversely associated with birth weight $(r=-0.24, p=0.01, n=109)$, custom
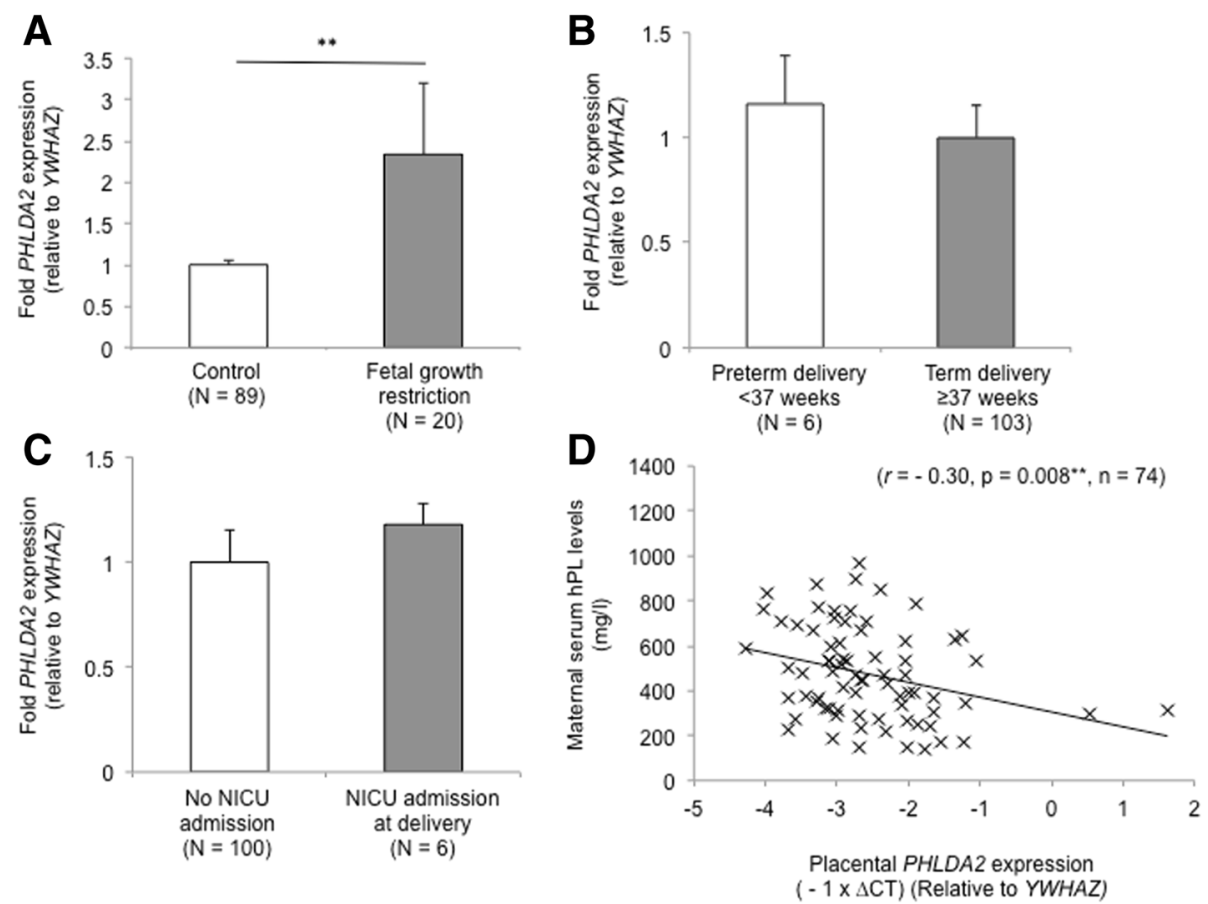

Fig. 1 Placental PHLDA2 expression in RFM pregnancies. Placental PHLDA2 expression was significantly increased in RFM pregnancies resulting in delivery of a growth restricted infant (a) Fetal growth restriction was defined as delivery of a term infant with a custom birth weight centile $<10^{\text {th }}$. Placental PHLDA2 was not significantly altered in RFM pregnancies where infants were born preterm (b) or admitted to NICU at delivery (c) There was a significant inverse association between placental PHLDA2 expression and maternal serum hPL levels (d) RFM = reduced fetal movements. Error bars represent SEM. ** $P<0.01$ 
birth weight centiles $(r=-0.25, p=0.01, n=109)$ and, in this study, also placental weight $(r=-0.39, p=0.03$, $n=31)$.

We subsequently analysed placental PHLDA2 expression in relation to preterm delivery and NICU admission at birth, which are other common poor perinatal outcomes associated with RFM. There was no significant correlation between placental PHLDA2 expression and gestational age $(r=-1.41, p=0.14, n=109)$ and was no significant difference in expression between preterm and term deliveries (Fig. 1b). Placental PHLDA2 expression was not significantly altered in infants admitted to NICU at delivery (for perinatal asphyxia) (Fig. 1c) and there was no significant correlation between placental PHLDA2 and other measures of infant wellbeing at delivery including Apgar scores at $1 \mathrm{~min}(r=0.01, p=0.93, n=$ $106)$ and $5 \min (r=-0.11, p=0.27, n=105)$ or with arterial cord blood $\mathrm{pH}(r=-0.7, p=0.52, n=76)$.

Imprinted genes have been demonstrated to regulate the endocrine lineage of the mouse placenta, in particular expression of placental lactogens [18]. In this study there was a significant inverse association between placental PHLDA2 expression and maternal serum hPL levels (Fig. 1d) suggesting that PHLDA2 may regulate the production of placental hormones in human pregnancies. In further multiple linear regression analysis controlling for infant birth weight, offspring gender and gestational age $\left(\mathrm{F}(4,69)=5.34, p=0.001, R^{2}=0.24\right)$, the association between placental PHLDA2 expression and serum hPL levels failed to reach statistical significance $(p=0.07)$, with only infant birth weight significantly associated with maternal serum hPL levels $(p=0.01)$. This is perhaps not surprising since, in the mouse model, elevated Phlda2 drives both growth restriction and reduced expression of placental lactogens [19, 21]. Data from the animal model supports a causal association between placental PHLDA2 expression and maternal serum hPL levels.

\section{Discussion}

A number of studies have demonstrated abnormally elevated placental PHLDA2 in pregnancies complicated by FGR (reviewed in [16]). The Phlda2 overexpression mouse model exhibits FGR and impaired placental development supporting a causal role for elevated PHLDA2 in human FGR $[19,20]$. Uniquely, this study examined a group of women reporting RFM, a group who are at high risk of poor perinatal outcomes. Within this group, there was a significant association between increased placental PHLDA2 expression and FGR. Moreover, the highest levels of PHLDA2 expression were present in the most severely growth restricted cases which also had the most severe placental phenotype [12]. Placental weight was also significantly inversely associated with PHLDA2 expression, which has not previously been reported. This is of clinical interest as RFM is thought to represent a fetal adaptation to prolonged placental insufficiency which, if undetected, may result in stillbirth [8]. While all participants in the current study delivered live born infants, increased expression of PHLDA2 has previously been reported in cases of spontaneous miscarriage or fetal death [26] and more specifically, in cases of fetal death attributed to FGR [27]. These results further highlight a role for placental PHLDA2 in poor perinatal outcomes.

Our second key finding in this study was the significant association between elevated placental PHLDA2 expression and decreased maternal hPL serum levels. After controlling for birth weight, this association failed to reach statistical significance $(p=0.07)$ but this can be explained if, as in the mouse $[19,21]$, elevated PHLDA2 drives both growth restriction and reduced placental lactogen expression. hPL plays an important role in maternal glucose management during pregnancy and in the control of fetal growth [28]. This relationship between placental PHLDA2 expression and placental hPL production would therefore be of considerable interest in understanding the mechanisms driving poor growth in utero.

\section{Conclusion}

This study demonstrated significantly elevated PHLDA2 expression in placentas of RFM pregnancies resulting in delivery of a growth restricted infant and identified an association between placental PHLDA2 expression and serum hPL levels. It has previously been suggested that PHLDA2 expression in the placenta could be used to postnatally identify types of fetal growth restriction and thus inform subsequent infant care [16]. Identifying hormonal biomarkers of abnormal placental PHLDA2 expression during pregnancy could be further used as a prenatal diagnostic tool to identify RFM pregnancies at risk of poor perinatal outcomes. This is of particular importance in the context of a recent UK report highlighting that more appropriate, better informed management of RFM could have prevented one third of the stillbirth cases examined [29].

\section{Availability of data and materials}

Cohort data and details on availability of material $[2,9]$.

\section{Additional file}

\footnotetext{
Additional file 1: Figure 1. Placental PHLDA2 expression in RFM pregnancies, normalised to the geometric mean of YWHAZ and L19 expression. PHLDA2 expression was significantly increased in placenta from pregnancies complicated by fetal growth restriction when normalized to YWHAZ expression (A) or to the geometric mean of $Y W H A Z$ and $L 19$ expression (B) in a subset of samples from the full cohort $(N=37)$. Expression of the housekeeping genes YWHAZ and $L 19$ was significantly correlated ( $r=0.94, p<0.001, n=37)$. There was no significant difference in expression between RFM placenta from normal birth weight pregnancies and pregnancies resulting in fetal growth restriction ( $p=0.37$ and $p=0.45$ respectively, $n=3$ (TIF $2931 \mathrm{~kb}$ )
} 


\section{Abbreviations}

FGR: feta growth restriction; hPL: human placental lactogen;

PHLDA2: Pleckstrin homology-like domain family A member 2; RFM: reduced fetal movements.

\section{Competing interests}

The authors declare that they have no competing interests.

\section{Authors' contributions}

$A B J, A E P H$ and RMJ conceived the project. AEPH was involved in the collection of samples and clinical data for the cohort. ABJ and SJT carried out the laboratory and statistical analysis. ABJ, SJT, AEPH and RMJ wrote the manuscript and all authors have read and approved the final version of the manuscript.

\section{Acknowledgements}

The authors would like to acknowledge all the women who participated in this study and the midwives at St Mary's Hospital (Manchester) for their help with sample collection. RFM cohort was supported by Manchester NIHR Biomedical Research. ABJ was supported by a BBSRC DTG studentship and subsequently MRC project grant MR/M013960/1. SJT was supported by BBSRC project grant BB/J015156/1.

\section{Author details}

'Cardiff School of Biosciences, Cardiff University, Cardiff, Wales CF10 3AX, UK. ${ }^{2}$ Maternal and Fetal Health Research Centre, University of Manchester, Manchester, UK.

\section{Received: 27 November 2015 Accepted: 26 February 2016} Published online: 05 March 2016

\section{References}

1. O'Sullivan O, Stephen G, Martindale E, Heazell AE. Predicting poor perinatal outcome in women who present with decreased fetal movements. J Obstet Gynaecol. 2009;29(8):705-10. doi:10.3109/01443610903229598.

2. Dutton PJ, Warrander LK, Roberts SA, Bernatavicius G, Byrd LM, Gaze D, Kroll J, Jones RL, Sibley CP, Froen JF, Heazell AE . Predictors of poor perinatal outcome following maternal perception of reduced fetal movements-a prospective cohort study. PLoS One. 2012;7(7):e39784. doi:10.1371/journal.pone.0039784

3. Holm Tveit JV, Saastad E, Stray-Pedersen B, Bordahl PE, Froen JF. Maternal characteristics and pregnancy outcomes in women presenting with decreased fetal movements in late pregnancy. Acta Obstet Gynecol Scand. 2009;88(12):1345-51. doi:10.3109/00016340903348375.

4. Winje BA, Saastad E, Gunnes N, Tveit JV, Stray-Pedersen B, Flenady V, Froen JF. Analysis of 'count-to-ten' fetal movement charts: a prospective cohort study. Bjog. 2011;118(10):1229-38. do:10.1111/j.1471-0528.2011.02993.x.

5. Sinha D, Sharma A, Nallaswamy V, Jayagopal N, Bhatti N. Obstetric outcome in women complaining of reduced fetal movements. J Obstet Gynaecol. 2007;27(1):41-3. doi:10.1080/01443610601016909.

6. Heazell AE, Sumathi GM, Bhatti NR. What investigation is appropriate following maternal perception of reduced fetal movements? J Obstet Gynaecol. 2005;25(7):648-50. doi:10.1080/01443610500278303.

7. Saastad E, Ahlborg T, Frøen JF. Low maternal awareness of fetal movement is associated with small for gestational age infants. J Midwifery Womens Health. 2008;53(4):345-52. doi:10.1016/j.jmwh.2008.03.001.

8. Warrander LK, Heazell AE. Identifying placental dysfunction in women with reduced fetal movements can be used to predict patients at increased risk of pregnancy complications. Med Hypotheses. 2011;76(1):17-20. doi:10.1016/.jmehy.2010.08.020

9. Warrander LK, Batra G, Bernatavicius G, Greenwood SL, Dutton P, Jones RL, Sibley CP, Heazell AE. Maternal perception of reduced fetal movements is associated with altered placental structure and function. PLoS One. 2012; 7(4), e34851. doi:10.1371/journal.pone.0034851.

10. Winje BA, Roald B, Kristensen NP, Froen JF. Placental pathology in pregnancies with maternally perceived decreased fetal movement-a population-based nested case-cohort study. PLoS One. 2012;7(6):e39259. doi:10.1371/journal.pone.0039259.

11. Pagani G, D'Antonio F, Khalil A, Papageorghiou A, Bhide A, Thilaganathan B. Association between reduced fetal movements at term and first trimester markers of impaired placental development. Placenta. 2014;35(8):606-10. doi:10.1016/j.placenta.2014.04.020.
12. Higgins LE, Rey de Castro N, Addo N, Wareing M, Greenwood SL, Jones RL, Sibley CP, Johnstone ED, Heazell AE. Placental features of late-onset adverse pregnancy outcome. PLoS One. 2015;10(6):e0129117. doi:10.1371/journal. pone.0129117.

13. Gude NM, Roberts CT, Kalionis B, King RG. Growth and function of the normal human placenta. Thromb Res. 2004;114(5-6):397-407. doi:10.1016/j.thromres. 2004.06.038.

14. Surani MA. Imprinting and the initiation of gene silencing in the germ line. Cell. 1998;93(3):309-12.

15. Tunster $\mathrm{SJ}$, Jensen $\mathrm{AB}$, John RM. Imprinted genes in mouse placental development and the regulation of fetal energy stores. Reproduction. 2013; 145(5):R117-137. doi:10.1530/rep-12-0511.

16. Jensen AB, Tunster SJ, John RM. The significance of elevated placental PHLDA2 in human growth restricted pregnancies. Placenta. 2014;35(8):528-32. doi:10.1016/.jplacenta.2014.04.018

17. Cleaton MA, Edwards CA, Ferguson-Smith AC. Phenotypic outcomes of imprinted gene models in mice: elucidation of pre- and postnatal functions of imprinted genes. Annu Rev Genomics Hum Genet. 2014;15:93-126. doi:10.1146/annurev-genom-091212-153441.

18. John RM. Epigenetic regulation of placental endocrine lineages and complications of pregnancy. Biochem Soc Trans. 2013;41(3):701-9. doi:10. 1042/bst20130002

19. Tunster SJ, Tycko B, John RM. The imprinted Phlda2 gene regulates extraembryonic energy stores. Mol Cell Biol. 2010;30(1):295-306.

20. Tunster SJ, Van De Pette M, John RM. Isolating the role of elevated Phlda2 in asymmetric late fetal growth restriction in mice. Dis Model Mech. 2014; 7(10):1185-91. doi:10.1242/dmm.017079.

21. Tunster SJ, Creeth HD, John RM. The imprinted Phlda2 gene modulates a major endocrine compartment of the placenta to regulate placental demands for maternal resources. Dev Biol. 2016;409(1):251-60. doi:10.1016/j. ydbio.2015.10.015

22. Janssen AB, Tunster SJ, Savory N, Holmes A, Beasley J, Parveen SA, Penketh RJ, John RM. Placental expression of imprinted genes varies with sampling site and mode of delivery. Placenta. 2015;36(8):790-5. doi:10.1016/jplacenta.2015.06.011.

23. Apostolidou S, Abu-Amero S, O'Donoghue K, Frost J, Olafsdottir O, Chavele K, Whittaker J, Loughna P, Stanier P, Moore G. Elevated placental expression of the imprinted PHLDA2 gene is associated with low birth weight. J Mol Med. 2007:85(4):379-87. doi:10.1007/s00109-006-0131-8.

24. O'Donnell KJ, Bugge Jensen A, Freeman L, Khalife N, O'Connor TG, Glover V. Maternal prenatal anxiety and downregulation of placental 11 beta-HSD2. Psychoneuroendocrinology. 2012;37(6):818-26. doi:10.1016/j.psyneuen.2011.09.014.

25. Livak KJ, Schmittgen TD. Analysis of relative gene expression data using real-time quantitative PCR and the 2(-Delta Delta C(T)) Method. Methods. 2001;25(4):402-8. doi:10.1006/meth.2001.1262

26. Doria S, Sousa M, Fernandes S, Ramalho C, Brandao O, Matias A, Barros A Carvalho F. Gene expression pattern of IGF2, PHLDA2, PEG10 and CDKN1C imprinted genes in spontaneous miscarriages or fetal deaths. Epigenetics. 2010;5(5):444-50.

27. Cordeiro A, Neto AP, Carvalho F, Ramalho C, Doria S. Relevance of genomic imprinting in intrauterine human growth expression of CDKN1C, H19, IGF2, KCNQ1 and PHLDA2 imprinted genes. J Assist Reprod Genet. 2014;31(10): 1361-8. doi:10.1007/s10815-014-0278-0.

28. Newbern D, Freemark M. Placental hormones and the control of maternal metabolism and fetal growth. Curr Opin Endocrinol Diabetes Obes. 2011; 18(6):409-16. doi:10.1097/MED.0b013e32834c800d.

29. Draper E, Kurinczuk J, Kenyon S, on behalf of MBRRACE-UK. MBRRACE-UK Perinatal Confidential Enquiry: Term, singleton, normally formed, antepartum stillbirth. Leicester: The Infant Mortality and Morbidity Studies, Department of Health Sciences, University of Leicester; 2015. 